\title{
Univariate and Bivariate Empirical Mode Decomposition for Postural Stability Analysis
}

\author{
Hassan Amoud, Hichem Snoussi, David Hewson, and Jacques Duchêne \\ Charles Delaunay Institute, FRE CNRS 2848, University of Technology of Troyes, 10000 Troyes, France \\ Correspondence should be addressed to Hassan Amoud, hassan.amoud@utt.fr
}

Received 22 October 2007; Accepted 7 February 2008

Recommended by Kenneth Barner

The aim of this paper was to compare empirical mode decomposition (EMD) and two new extended methods of EMD named complex empirical mode decomposition (complex-EMD) and bivariate empirical mode decomposition (bivariate-EMD). All methods were used to analyze stabilogram center of pressure (COP) time series. The two new methods are suitable to be applied to complex time series to extract complex intrinsic mode functions (IMFs) before the Hilbert transform is subsequently applied on the IMFs. The trace of the analytic IMF in the complex plane has a circular form, with each IMF having its own rotation frequency. The area of the circle and the average rotation frequency of IMFs represent efficient indicators of the postural stability status of subjects. Experimental results show the effectiveness of these indicators to identify differences in standing posture between groups.

Copyright (C) 2008 Hassan Amoud et al. This is an open access article distributed under the Creative Commons Attribution License, which permits unrestricted use, distribution, and reproduction in any medium, provided the original work is properly cited.

\section{INTRODUCTION}

Falls are a major problem for the elderly due to the resulting loss of autonomy and subsequent behavior modification related to fear of falling. Health care services worldwide are actively working on the issue of falls for both medical and social reasons. For instance, in France alone, the number of deaths attributed annually to falls is estimated to be more than 9000, with a resultant cost in excess of two billion euros [1]. The cost of falls is only going to increase, in line with the increase in the elderly population. In Europe, the percentage of adults over 65 years old will almost double from $15 \%$ to $30 \%$ by the year 2050 [2]. Falling is a consequence of a failure in the postural control system due to aging or a specific pathology. Many risk factors have been identified for falls, the most commonly cited including an underlying muscular weakness, a previous fall, as well as balance and gait problems [3].

Postural equilibrium is maintained by reacting to information from the different sensory systems, including vestibular, visual, and proprioception systems. It is possible to evaluate the postural control system using either clinical or biomechanical tests. Biomechanical tests can be dynamic or static, with dynamic tests used to characterize the per- formance of the postural control system to maintain the posture after an external perturbation. In contrast, static tests evaluate postural performance in a static position. In static posture, a force plate is used to evaluate postural sway. The force plate measures the displacement of the center of pressure (COP), which represents the location of the resultant force exerted on the surface of a force plate. The COP, which can be used as a measure of postural stability, is measured in the horizontal plane in both anteroposterior (AP) and mediolateral (ML) directions [4]. The representation of the COP time series in AP and ML directions is known as the stabilogram (see Figure 1).

Traditional parameters are extracted from the stabilogram signals under the assumption that the COP is a stationary time series $[4,5]$. These classical parameters give few insights into the control of posture [6], providing purely statistical information while ignoring the dynamic characteristics of COP displacements. These parameters include temporal (mean, RMS), spatiotemporal (area of the ellipse), and spectral (median frequency) parameters.

Recently, parameters that describe the fractal and time evolutionary properties of the $\mathrm{COP}$ and provide information related to underlying physiological control processes have been extracted using nonlinear and fractal analyses. These 


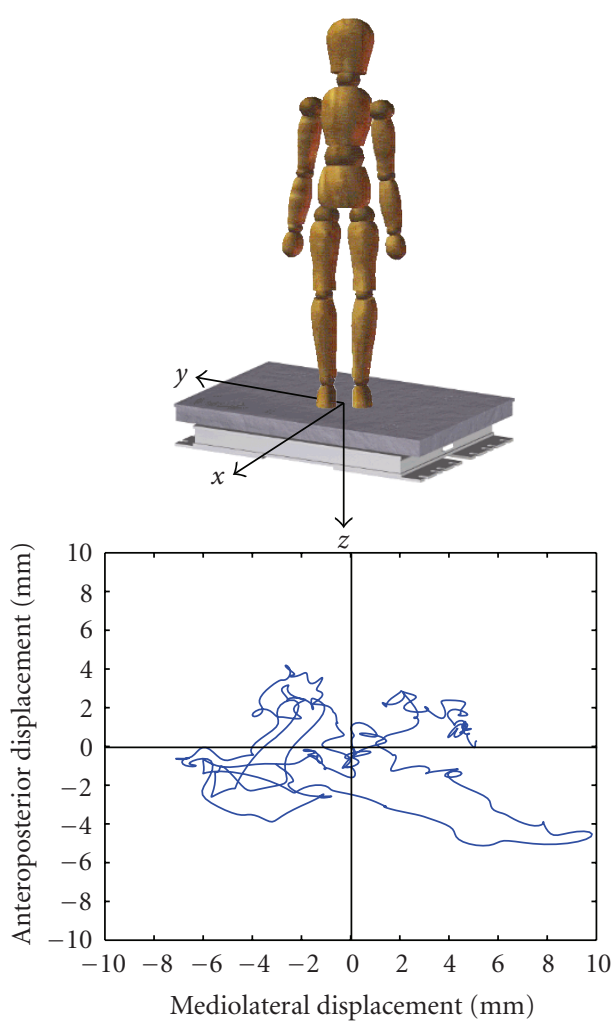

(a)
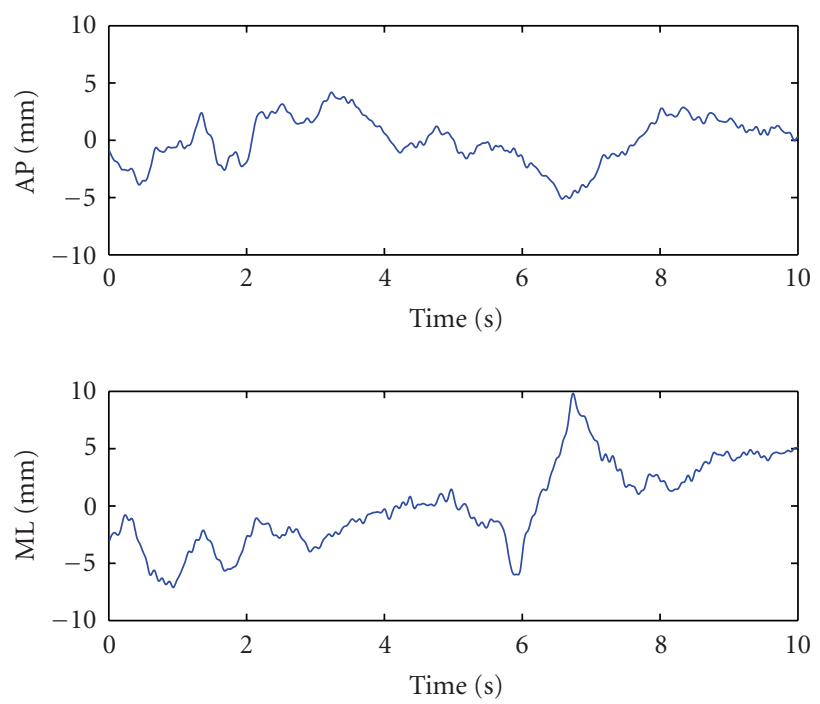

(b)

Figure 1: Displacement of the center of pressure in the horizontal plane (bottom left), displacement in the anteroposterior direction over time (top right), and displacement in the mediolateral direction over time (bottom right). Data are recorded from a healthy control subject for 10 seconds.

new groups of parameters include the Hurst exponent, which provides information about long-term correlation [7], the reconstructed phase space [8], and entropy [9].

Stabilogram signals have been shown to be nonstationary signals $[10,11]$. In order to extract information from the stabilogram and to characterize the postural stability, it is proposed in this paper to apply the Hilbert transform on the intrinsic mode functions (IMFs) extracted by the empirical mode decomposition (EMD) [12]. The EMD method decomposes the signal into a set of IMFs which represent the oscillatory modes embedded in the signal. For the COP signal, IMFs have well-defined instantaneous frequency and different IMFs do not exhibit the same frequency at the same time. In addition, the COP signal is a bidirectional signal and can be represented by a complex signal $(\mathrm{ML}+j \mathrm{AP})$. The two components of the COP signal are nonlinearly correlated due to the complex correlation between all the postural control systems. To analyze the complex COP signal, two new methods derived from EMD method to decompose a complex signal will be applied. The first method, complex empirical mode decomposition (complex-EMD), was developed in [13], while the second method, bivariate empirical mode decomposition (bivariateEMD) was developed in [14, 15].

The complex-EMD method is based on the inherent relationship between the positive and negative frequency components of a complex signal. This method treats the positive and negative frequency components as two independent signals. The EMD of these two components gives two sets of IMFs corresponding to the positive and negative frequency components of a complex signal. The bivariate-EMD method is based on the idea of replacing the oscillation notion in EMD by a notion of rotation. This method considers that the bivariate signal can be described as the sum of a fast rotation component superimposed on a slower rotation. The bivariate-EMD algorithm consists in projecting the complex signal on a set of directions and then applying the sifting process of the basic-EMD on the projected components.

The EMD, complex-EMD, and bivariate-EMD methods are powerful signal processing techniques that can be used to analyze the stabilogram and to characterize the quality of equilibrium in standing posture. In fact, the plots of the analytic IMFs in the complex plane have a specific geometry similar to a circular form; and each IMF has its own rotation frequency. In contrast, the analytic signal of the entire stabilogram signal does not have a specific form, and it differs between experiments for the same subject due to its inherent nonstationary nature. Furthermore, the stabilogram is a multicomponent signal, thus its instantaneous frequency cannot be calculated directly. These two features (circular form and rotation frequency) permit the extraction of new nonlinear parameters that characterize the quality of equilibrium and provide more information about the mechanisms underlying postural control. The first parameter is simply the area of the circle formed by the shape of the analytic IMF, while the second parameter is the average rotation frequency.

In order to test the capacity of different EMD methods and the Hilbert transform to describe the quality of equilibrium and to discriminate between elderly and control group, the three EMD methods were applied on stabilogram signals extracted from elderly and control groups. The extracted nonlinear parameters from the plots of the analytic IMFs were the area of the circle in the complex plane, and the 


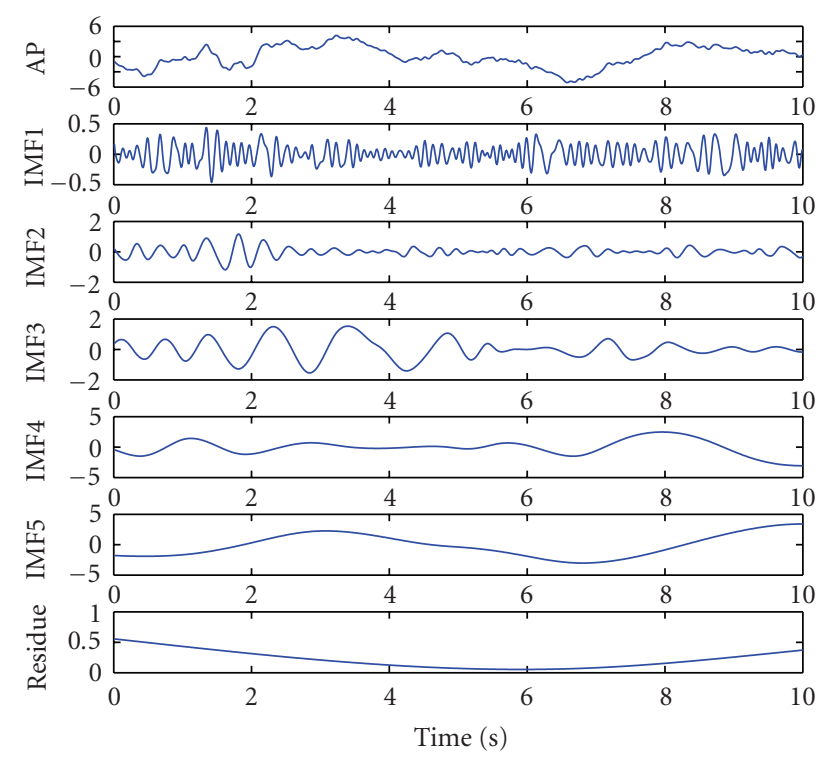

FIGURE 2: EMD decomposition of the 10-second anteroposterior displacement time series in Figure 1.

average rotation frequency of each IMF. The paper is organized as follows. The experimental protocol, EMD, complexEMD, bivariate-EMD, and the parameter calculation are presented in Section 2. The results of the application of the cited methods on the stabilogram are presented in Section 3. Discussions about the results and the EMD methods are presented in Section 4.

\section{METHODS}

\subsection{Subjects}

Ten healthy control subjects (three males and seven females) and ten healthy elderly subjects (four males and six females) participated in the study. Control subjects' mean age, height, and weight were $33.3 \pm 7.4 \mathrm{y}, 168.0 \pm 6.5 \mathrm{~cm}$, and $65.7 \pm$ $17.6 \mathrm{~kg}$, respectively. Elderly subjects' mean age, height and weight were $80.5 \pm 4.7 \mathrm{y}, 165.6 \pm 7.0 \mathrm{~cm}$, and $71.9 \pm 9.9 \mathrm{~kg}$, respectively. All subjects who participated gave their written informed consent. No subjects reported any musculoskeletal or neurological conditions that precluded their participation in the study.

\subsection{Data acquisition and data processing}

Center of pressure data were obtained from a Bertec 406008 force plate (Bertec Corporation, Columbus, Ohio, USA). The initial COP signals were calculated with respect to the center of the force plate before normalization by subtraction of the mean value. Data were recorded using ProTags and developed in Labview (National Instruments Corporation, Austin, Tex, USA). Data were sampled at $100 \mathrm{~Hz}$, using an 8th-order lowpass Butterworth filter with a cutoff frequency of $10 \mathrm{~Hz}$. All subsequent calculations were performed using MATLAB (Mathworks Inc, Natick, Mass, USA).

\subsection{Experimental protocol}

Subjects were tested barefoot or wearing socks. Testing began with subjects standing upright with their arms by their sides in front of the force-plate while looking at a 10$\mathrm{cm}$ cross fixed on the wall two meters in front of them. Upon verbal instruction, subjects stepped onto the forceplate. Subjects were not required to use a preordained foot position. Data recording lasted 15 seconds, during which time subjects maintained an upright posture. A second verbal command was given for subjects to step down from the force plate.

\subsection{Empirical mode decomposition}

EMD is a signal processing decomposition technique that decomposes the signal into waveforms modulated in both amplitude and frequency by extracting all of the oscillatory modes embedded in the signal [12]. The decomposition is an intuitive and adaptive signal-dependent decomposition and does not require any conditions about the stationarity and linearity of the signal. The waveforms extracted by EMD are named IMFs. Each IMF is symmetric, is assumed to yield a meaningful local frequency, and different IMFs do not exhibit the same frequency at the same time. In other words, each IMF satisfies the two following constraints:

(i) the number of extrema and the number of zero crossings are identical or differ at most by one;

(ii) the mean value between the upper and the lower envelope is equal to zero at any time.

The difference between the original signal and the IMF time series is the residual. The first IMF component is obtained by a sifting process. This procedure is then applied on the residual in order to extract the second IMF, and so forth. Thus all the IMFs are iteratively extracted. The nonstationary signal $x(t)$ is then represented as a linear sum of IMFs and the residual component:

$$
x(t)=\sum_{k=1}^{K} d_{k}(t)+r_{K}(t),
$$

where $d_{k}(t)$ denotes the $k$ th extracted empirical mode and $r_{K}(t)$ the residual.

The EMD algorithm can be summarized as follows.

(1) Extract all the extrema of $x(t)$.

(2) Interpolate between minima (resp., maxima) to obtain two envelopes $e_{\min }(t)$ and $e_{\max }(t)$.

(3) Compute the average: $m(t)=\left(e_{\min }(t)+e_{\max }(t)\right) / 2$.

(4) Extract the detail $d(t)=x(t)-m(t)$.

(5) Test if $d(t)$ is an IMF:

(i) if yes, repeat the procedure from the step 1 on the residual signal $r(t)=x(t)-d(t)$,

(ii) if not, replace $x(t)$ with $d(t)$ and repeat the procedure from step 1 . 


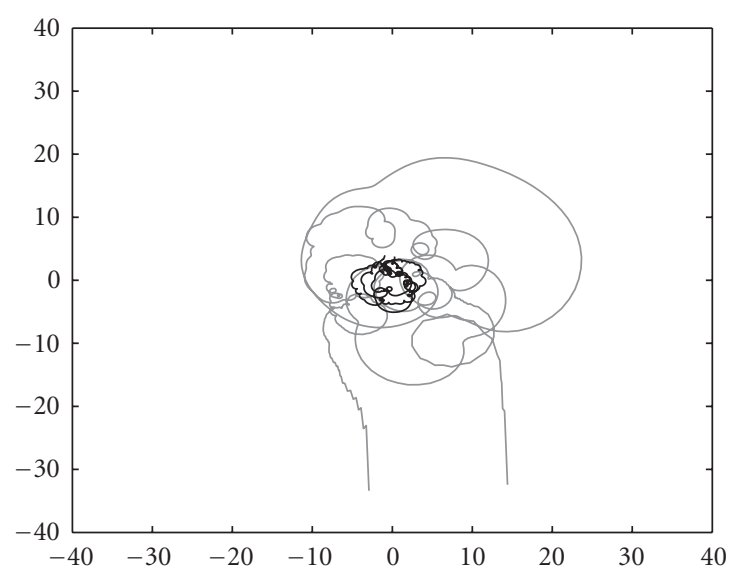

(a)

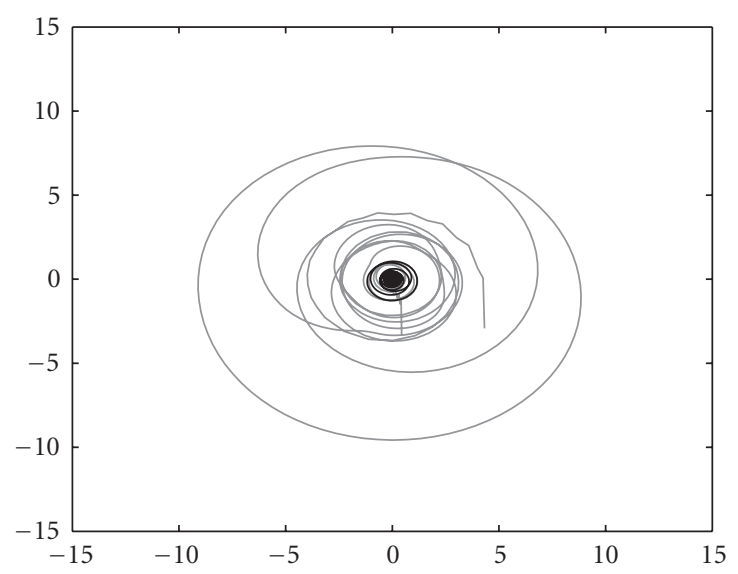

(c)

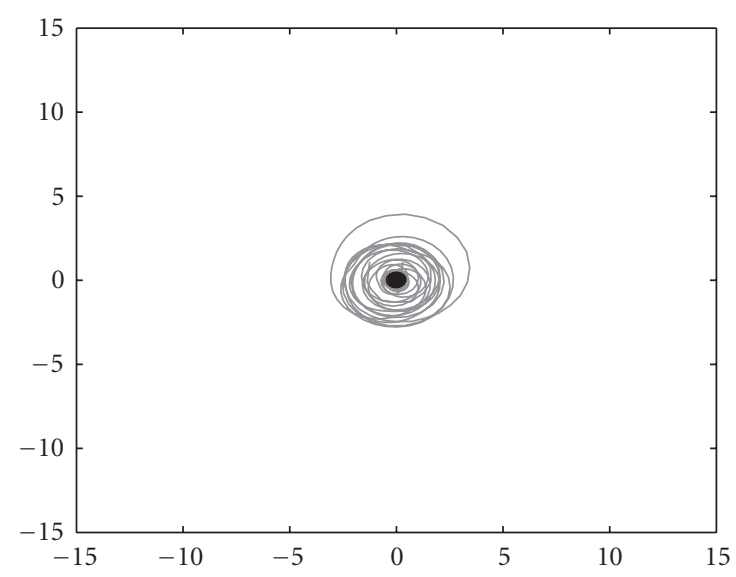

(b)

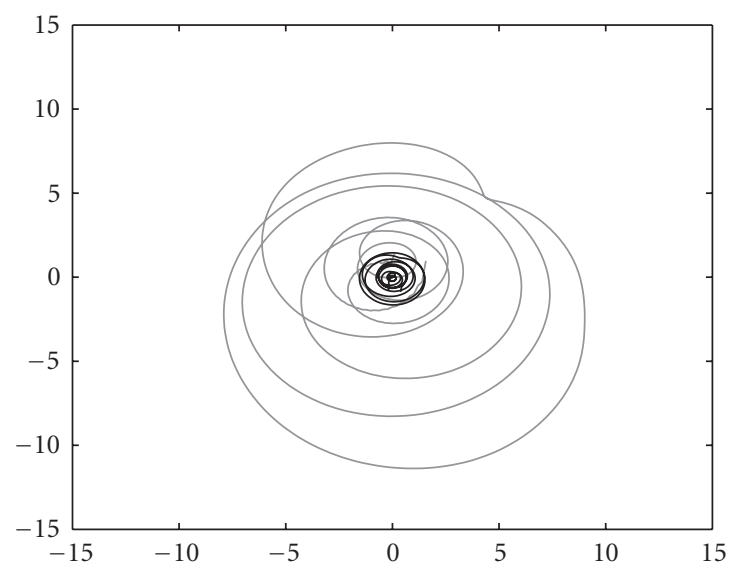

(d)

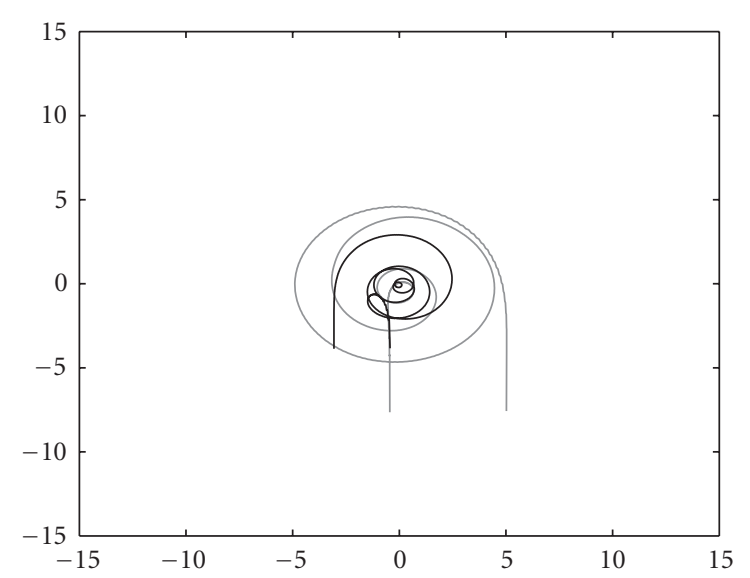

(e)

FIGURE 3: Representation in the complex plane of the analytic signal of a healthy control subject (black lines) and healthy elderly subject (grey lines) for the AP direction (a). Analytic signals of the first four IMFs (b): IMF1, (c): IMF2, (d): IMF3, and (e): IMF4.

Matlab codes are available at http://perso.ens-lyon.fr/ patrick.flandrin/emd.html.

An example of the application of EMD on the 10-second AP time series traced in Figure 1 is shown in Figure 2. The number of IMFs varied between experiments and between subjects. The minimum number of IMFs was 4 .

\subsection{Hilbert transformation and phase estimation}

The Hilbert transform of a real signal $x(t)$ is defined as

$$
y(t)=\frac{1}{\pi} \text { p.v. } \int_{-\infty}^{+\infty} \frac{x(\tau)}{t-\tau} d \tau
$$


where p.v. indicates the Cauchy principle value. The analytic signal of $x(t)$ is then defined as:

$$
z(t)=x(t)+j y(t) .
$$

The analytic signal can be further expressed as

$$
z(t)=a(t) \exp [j \theta(t)]
$$

where $a(t)=\sqrt{x^{2}(t)+y^{2}(t)}$ is the amplitude of $z(t)$, and $\theta(t)=\arctan y(t) / x(t)$ is the instantaneous phase from which the instantaneous signal frequency $f(t)$ is obtained by differentiation:

$$
f(t)=\frac{1}{2 \pi} \frac{\partial \theta(t)}{\partial t} .
$$

The original signal $x(t)$ is now expressed in a Fourier-like expansion as

$$
x(t)=\operatorname{Re}\left\{\sum_{k=1}^{K+1} a_{k}(t) \exp \left[j \int 2 \pi f_{k}(t) d t\right]\right\}
$$

in which the residual $r_{K}(t)$ is included and where the index $k$ refers to each IMF and $\operatorname{Re}\{\cdot\}$ denotes the real part of a complex quantity. Huang et al. proposed that the Hilbert transform should be applied on all IMFs obtained by EMD. This transform is known as Hilbert-Huang transform (HHT). Indeed, the IMFs are locally symmetric functions and therefore the instantaneous frequency is well localized in the time-frequency domain.

An example of the HHT (with scalar EMD) applied on two AP time series is presented in Figure 3. The first AP time series was recorded from a healthy control subject (black lines), while the second time series was recorded from a healthy elderly subject (grey lines). This figure presents the trace of the entire analytic signal in the complex plane (Figure 3(a)), as well as those of each analytic IMF extracted with the basic-EMD (Figures 3(b)-3(e)). It can be seen that the forms of these traces are similar to circles. A discussion about this figure is presented in the discussion section.

In Figure 4, the phase time series of each IMF and the average rotation frequencies of a control subject are presented. The average rotation frequency is equal to the slope estimate of the phase time series divided by $2 \pi$. It can be observed that the average rotation frequency decreases from the first IMF to the last IMF simply because the sifting procedure picks the component with the fastest variation embedded in the original signal first and that with the slowest variation last.

\subsection{Complex empirical mode decomposition}

The complex-EMD is an extension of the basic-EMD suitable for dealing with complex signals [13]. The motivation to extend EMD is that a large number of signal processing applications have complex signals. In addition, this extension is applied on both the real and imaginary parts simultaneously because complex signals have a mutual dependence between the real and imaginary parts. Thus, if the decomposition is done separately, the mutual dependency will be lost. The algorithm of the complex-EMD is as follows.

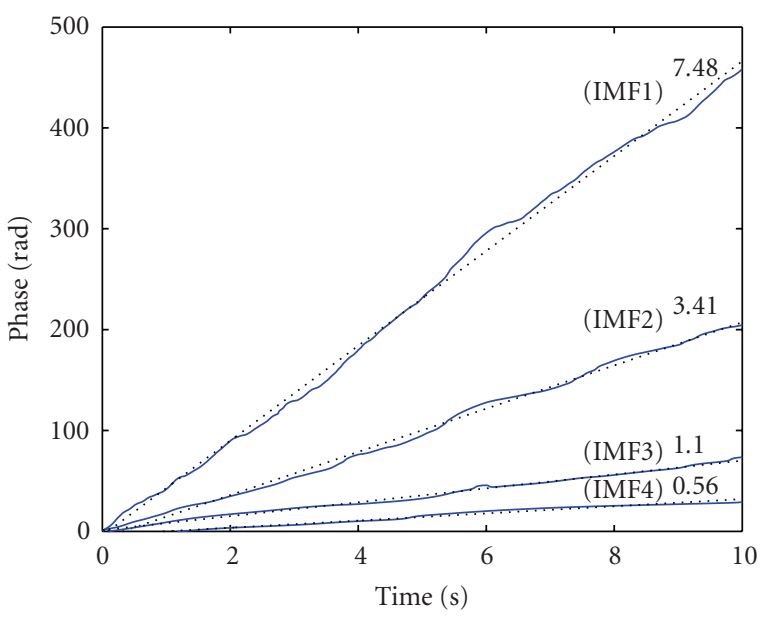

FIgUre 4: Traces of the instantaneous phase and the value of the average rotation frequency for the first four IMFs of the AP displacement traced in Figure 2.

(1) Extract the positive and negative frequency components to generate two analytic signals $X_{+}\left(e^{j w}\right)$ and $X_{-}\left(e^{j w}\right)$ as follows:

$$
\begin{aligned}
& X_{+}\left(e^{j w}\right)=H\left(e^{j w}\right) X\left(e^{j w}\right), \\
& X_{-}\left(e^{j w}\right)=H\left(e^{j w}\right) X^{*}\left(e^{-j w}\right),
\end{aligned}
$$

where $H\left(e^{j w}\right)$ is an ideal bandpass filter equal to one for $0 \leq w<\pi$ and zero for $-\pi \leq w<0, X^{*}\left(e^{j w}\right)$ is the complex conjugate of $X\left(e^{j w}\right)$.

(2) Extract the real part of the inverse Fourier transform of $X_{+}\left(e^{j w}\right)$ and $X_{-}\left(e^{j w}\right)$, denoted by $x_{+}(t)$ and $x_{-}(t)$.

(3) Apply the basic-EMD on $x_{+}(t)$ and $x_{-}(t)$ separately to extract the IMFs of the positive and negative components denoted by $\left\{x_{i}(t)\right\}_{i=1}^{N_{+}}$and $\left\{x_{i}(t)\right\}_{i=-N_{-}}^{-1}$.

Finally, $x_{+}(t)$ and $x_{-}(t)$ can be expressed as

$$
\begin{aligned}
& x_{+}(t)=\sum_{i=1}^{N_{+}} x_{i}(t)+r_{+}(t), \\
& x_{-}(t)=\sum_{i=-N_{-}}^{-1} x_{i}(t)+r_{-}(t),
\end{aligned}
$$

where $r_{+}(t)$ and $r_{-}(t)$ are the residuals of $x_{+}(t)$ and $x_{-}(t)$, respectively.

The complex-EMD can now be expressed as

$$
x(t)=\sum_{i=-N_{-}, i \neq 0}^{N_{+}} y_{i}(t)+r(t),
$$

where $y_{i}(t)=x_{i}(t)+j \mathscr{H}\left[x_{i}(t)\right]$, and $\mathscr{H}[\cdot]$ denotes the Hilbert transform operator.

\subsection{Bivariate empirical mode decomposition}

Bivariate-EMD is another extension of the EMD to complex signals. The main difference between the bivariate-EMD and 
the complex-EMD is that the latter uses the basic-EMD to decompose complex signals, whereas the bivariate-EMD adapts the rationale underlying the EMD to a bivariate framework $[14,15]$. The algorithm of the bivariate-EMD, as proposed in [14], is as follows:

(1) For $1 \leq m \leq M$,

(a) project $x(t)$ on direction $\phi_{m}: p_{\phi_{m}}(t)=$ $\operatorname{Re}\left(e^{-j \phi_{m}} x(t)\right)$

(b) extract the maxima of $p_{\phi_{m}}(t):\left(t_{i}^{m}, p_{i}^{m}\right)$,

(c) interpolate the set of points $\left(t_{i}^{m}, e^{j \phi_{m}} p_{i}^{m}\right)$ to obtain the partial envelope curve in direction $\phi_{m}$ named $e_{\phi_{m}}(t)$.

(2) Compute the mean of all tangents as follows: $e(t)=$ $(2 / M) \sum_{m} e_{\phi_{m}}(t)$.

(3) Subtract the mean to obtain $d(t)=x(t)-e(t)$.

(4) Test if $d(t)$ is an IMF:

(a) if yes, repeat the procedure from the step 1 on the residual signal,

(b) if not, replace $x(t)$ with $d(t)$ and repeat the procedure from step 1.

The bivariate-EMD can now be expressed as

$$
x(t)=\sum_{k} d_{k}(t)+r(t)
$$

where $d_{k}(t)$ denotes the $k$ th extracted complex empirical mode and $r(t)$ the residual.

\subsection{Parameter calculation}

Two types of parameters were calculated from the decomposition of COP signals into IMFs using the three methods of decomposition, EMD, complex-EMD, and bivariate-EMD. The first parameter is related to the form of the analytic IMFs in the complex plane, which can be seen to be circular. The parameter extracted is the area of the circle in which 95\% of the data points are located $\left(A r e a C_{\mathrm{IMF}}\right)$. This parameter was calculated for each of the first four IMFs. The second parameter, which is related to the phase of the analytic IMFs, is the average rotation frequency $\left(F_{\mathrm{IMF}}\right)$ of the analytic IMFs in the complex plane. These parameters were calculated for both AP and ML directions for the basic-EMD method and for ML $+j \mathrm{AP}$ for the complex-EMD and bivariateEMD methods. For each COP signal, a total of 24 areas and 24 frequencies were calculated using the three different methods. For the bivariate-EMD, we have used $M=4$ projections.

\subsubsection{Data analysis}

Center of pressure data were calculated from the moment the second foot contacted the force plate (FC2). FC2 was calculated as the time at which the maximum value of the second derivative of the ML signal occurred, which corresponded to the time the second foot touched the force plate. At this point, the largest acceleration of ML would occur when the COP moved rapidly towards the second foot. This instant in time was used for both AP and ML displacements. All analyses were performed for the 10 -second period starting 1 second after FC2, in order to give both AP and ML displacement time to return to near central values. The choice of FC2 has been validated in previous work [7]. Statistical analyses were performed with the statistical package for social sciences (SPSS Inc., Chicago, Ill, USA). The Kolmogorov-Smirnov test was used to check for normality. Owing to the grossly non-normal distribution of the area parameters $\left(A r e a C_{\mathrm{IMF}}\right)$, it was necessary to apply a $\log$ transformation to all $\mathrm{Area} \mathrm{C}_{\mathrm{IMF}}$ in order that an analysis of variance (ANOVA) could be performed. ANOVA was used to compare results between conditions, with $A$ rea $C_{\mathrm{IMF}}$ and $F_{\mathrm{IMF}}$ as the dependent variables and the subject group as the independent variable. Alpha level was set at $P<0.05$.

\section{RESULTS}

There was a significant difference in $A r e a C_{\text {IMF }}$ values between groups for all IMFs for all three methods (basic-EMD for AP and ML, complex-EMD for negative and positive components, and bivariate-EMD for real and imaginary parts) (Figure 5). The area was greater for elderly subjects then for control subjects. These increases in the values of Area $C_{\mathrm{IMF}}$ are indicative of degradation in the balance due to the effect of age on postural stability.

In respect to the average rotation frequency $\left(F_{\mathrm{IMF}}\right)$ parameters, significant differences were observed for the basic-EMD for AP displacement for both IMF1 and IMF2, with smaller values observed for elderly subjects (Figure 6(a)). In respect to ML displacement for basic-EMD, significant differences were observed between elderly and control subjects for IMF1, IMF2, and IMF3 (Figure 6(b)). For the bivariate-EMD method, significant differences were observed only for IMF3 for the real part corresponding to ML (Figure 6(d)), and for IMF1 for the imaginary part corresponding to AP (Figure 6(c)). However, for the complex-EMD, the average rotation frequency was significantly different for all IMFs for both positive and negative components (Figures 6(e), 6(f)). The rotation frequency was lower for elderly subjects then for the control subjects.

\section{DISCUSSION}

The first point to be addressed is whether the use of the EMD methodology followed by the Hilbert transform, as used in the present study, provides greater insight than simply applying the Hilbert transform to the whole signal, which also detected significant differences in surface area between groups. The response is related to the nature of the trace of the analytic signal of whole signal. It can be observed in Figure $3(\mathrm{a})$ that the analytic signal in the complex plane does not have a specific geometry, making it impossible to define the circle that encloses $95 \%$ of the data points. In addition, the form of the analytic trace differs between experiments for the same subject, as well as between subjects. Such a tracing is indicative of multiple centers of rotation, possibly due to the 


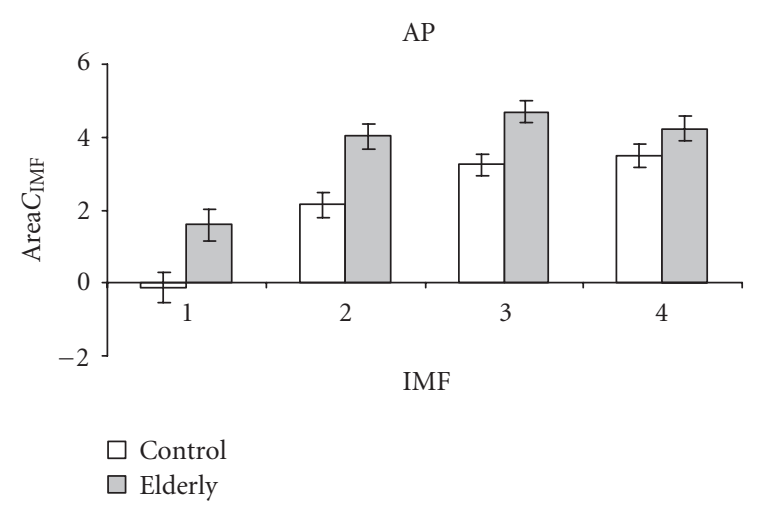

(a)

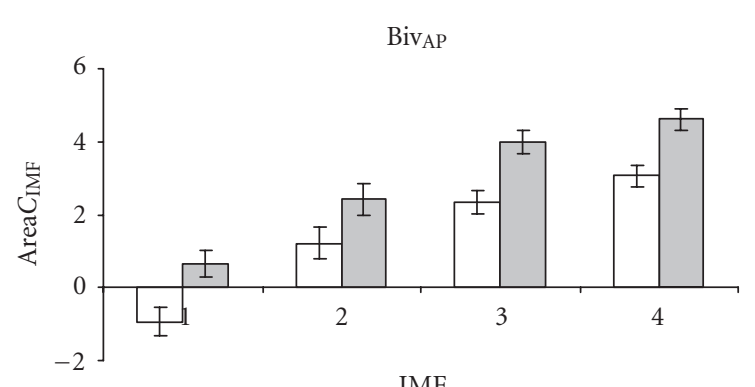

$\square$ Control

$\square$ Elderly

(c)

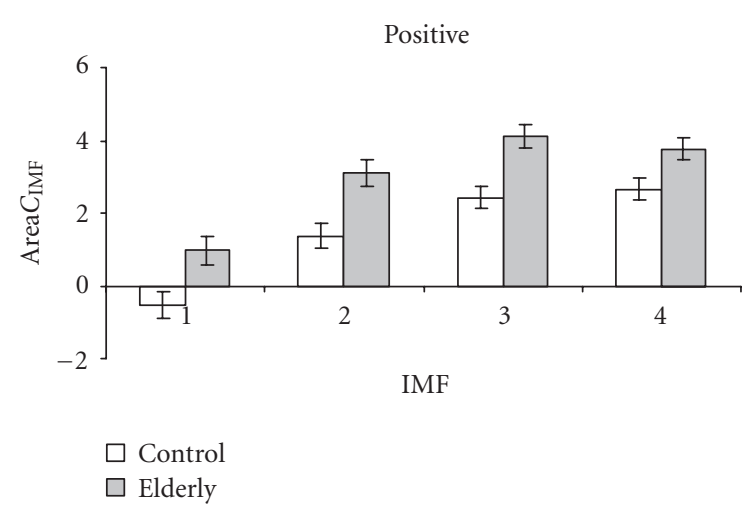

(e)

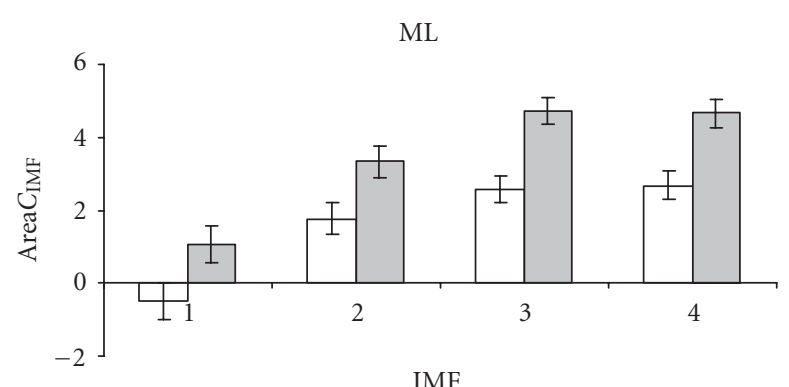

IMF

$$
\begin{aligned}
& \square \text { Control } \\
& \square \text { Elderly }
\end{aligned}
$$

(b)

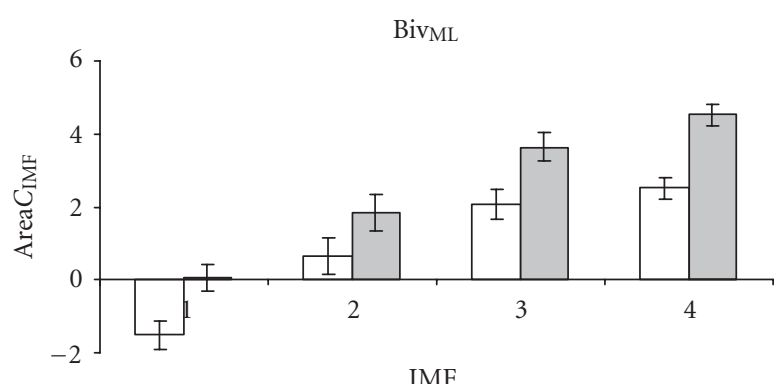

IMF

$$
\begin{aligned}
& \square \text { Control } \\
& \square \text { Elderly }
\end{aligned}
$$

(d)

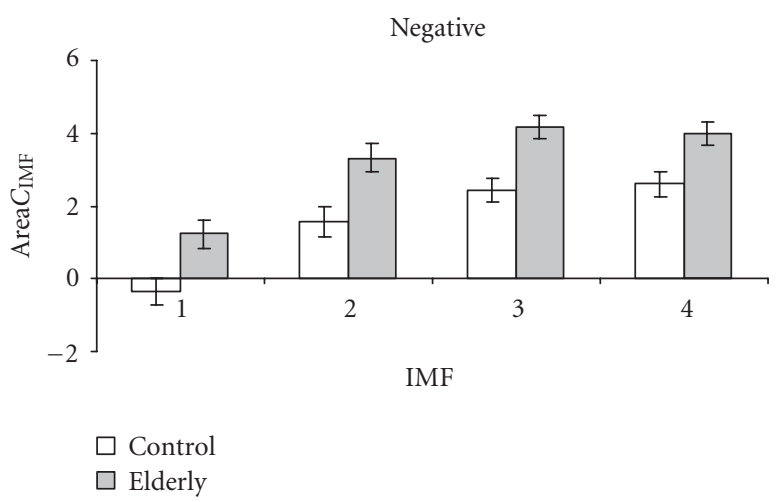

(f)

FIGURE 5: The surface area of the analytic signal in a logarithmic scale (Area $C_{\mathrm{IMF}}$ ) of the first four IMF for control and elderly subjects. (a) EMD for AP displacement; (b) EMD for ML displacement; (c) bivariate-EMD for the imaginary part of the signal (corresponding to AP displacement); (d) bivariate-EMD for the real part of the signal (ML); (e) complex-EMD for positive components; and (f) complex-EMD for negative components. Data are mean and 95\% confidence intervals. All comparisons are significantly different between groups.

presence of several different control strategies. In contrast, the traces of the analytic signals of each IMF always have a circular form (Figures 3(b)-3(e)), the area of which can then be estimated in the complex plane.

In respect to average rotation frequency, the trace of the phase as a function of time indicates a linear relation between phase and time. This relationship confirms the existence of a harmonic oscillation with a constant frequency equal to the average frequency. In addition, all the EMD methods permit the extraction of a proper rotation that could be related to different postural control systems.

In respect to the interpretation of the results for the postural data of the present study, elderly subjects had significantly greater surface areas for both AP and ML displacement than did the control subjects. The increased surface areas observed for the elderly subjects were due to the greater amplitude of postural sway for elderly subjects for all IMFs, thus indicating a less well-controlled posture. 
AP

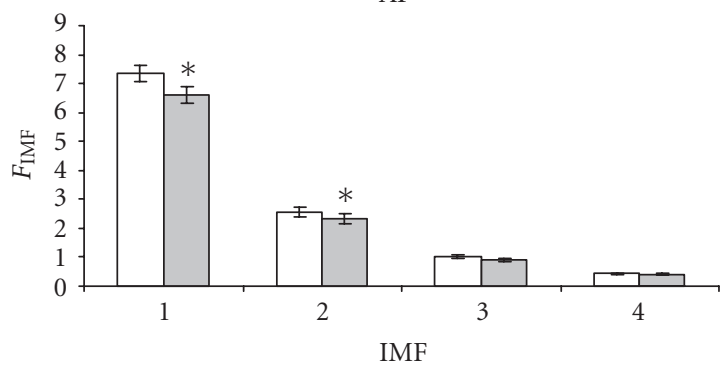

$\square$ Control

$\square$ Elderly

(a)

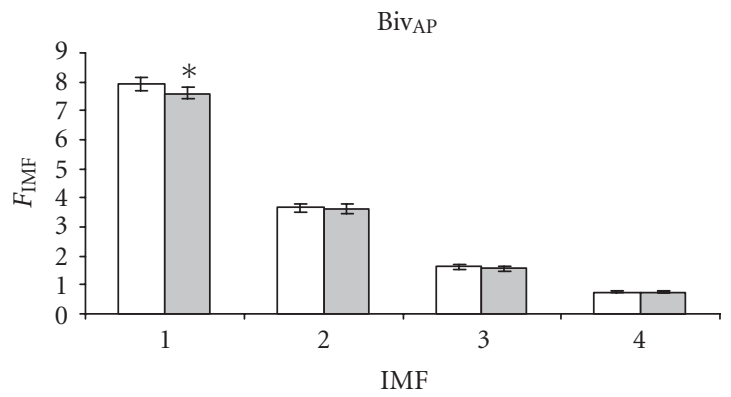

$\square$ Control

$\square$ Elderly

(c)

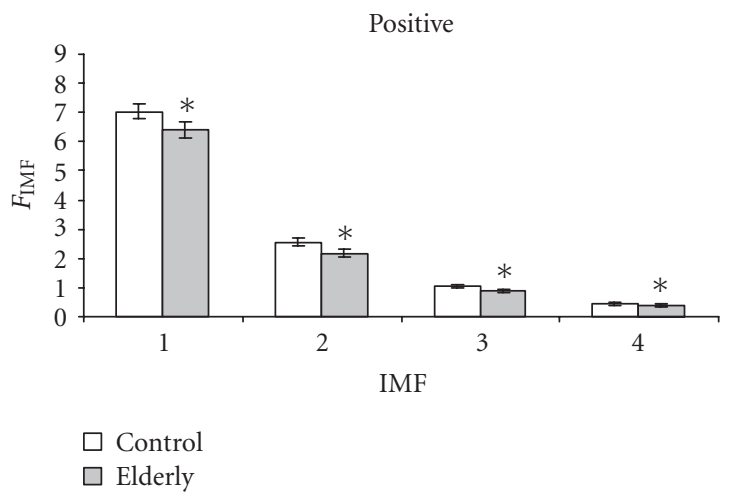

(e)
ML

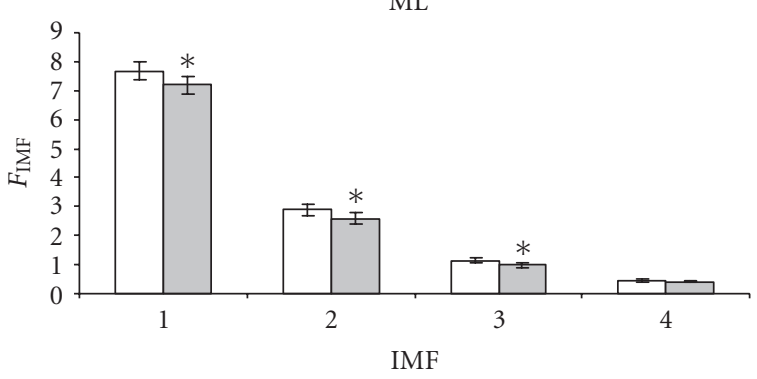

$$
\begin{aligned}
& \square \text { Control } \\
& \square \text { Elderly }
\end{aligned}
$$

(b)

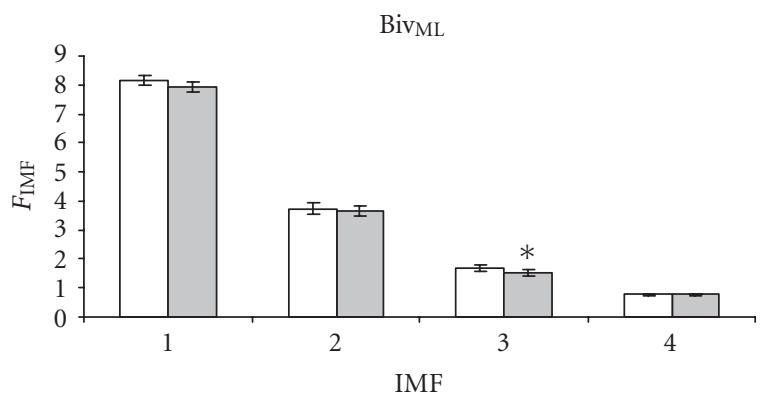

$$
\begin{aligned}
& \square \text { Control } \\
& \square \text { Elderly }
\end{aligned}
$$

(d)

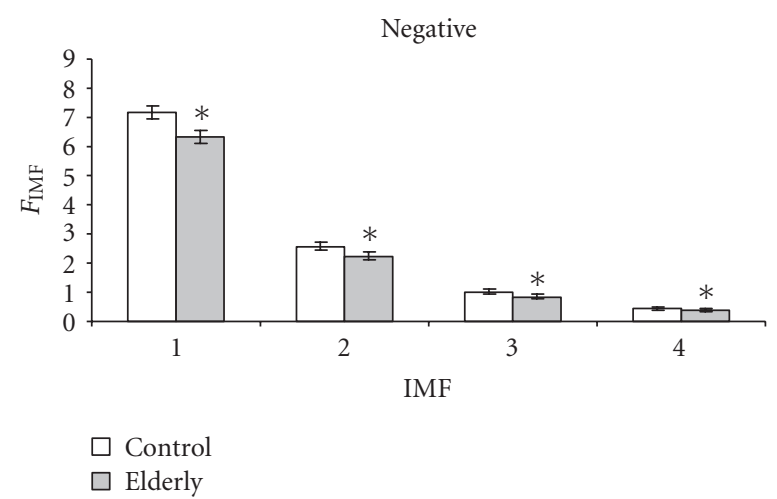

(f)

FIGURE 6: The average rotation frequency $\left(F_{\mathrm{IMF}}\right)$ of the first four IMF for control and elderly subjects. (a) EMD for AP displacement; (b) EMD for ML displacement; (c) bivariate-EMD for the imaginary part of the signal (corresponding to AP displacement); (d) bivariate-EMD for the real part of the signal (ML); (e) complex-EMD for positive components; and (f) complex-EMD for negative components. Data are mean and $95 \%$ confidence intervals. ${ }^{*}$ Significant difference from control subjects.

In respect to the rotation frequency, in contrast to surface area, smaller values were observed for elderly subjects. This contrast is self-explanatory, as surface area and rotation frequency are negatively correlated. Given that the timeseries length is the same for the both groups, it follows that if the radius of the circle is greater, the rotation frequency will be smaller.

\subsection{Comparison between one-dimensional and two-dimensional EMD methods}

For the one-dimensional EMD method, the mutual dependence between the real and imaginary parts of the complex signal is lost and is mapped onto two real independent signals that are decomposed separately. In the case of the 

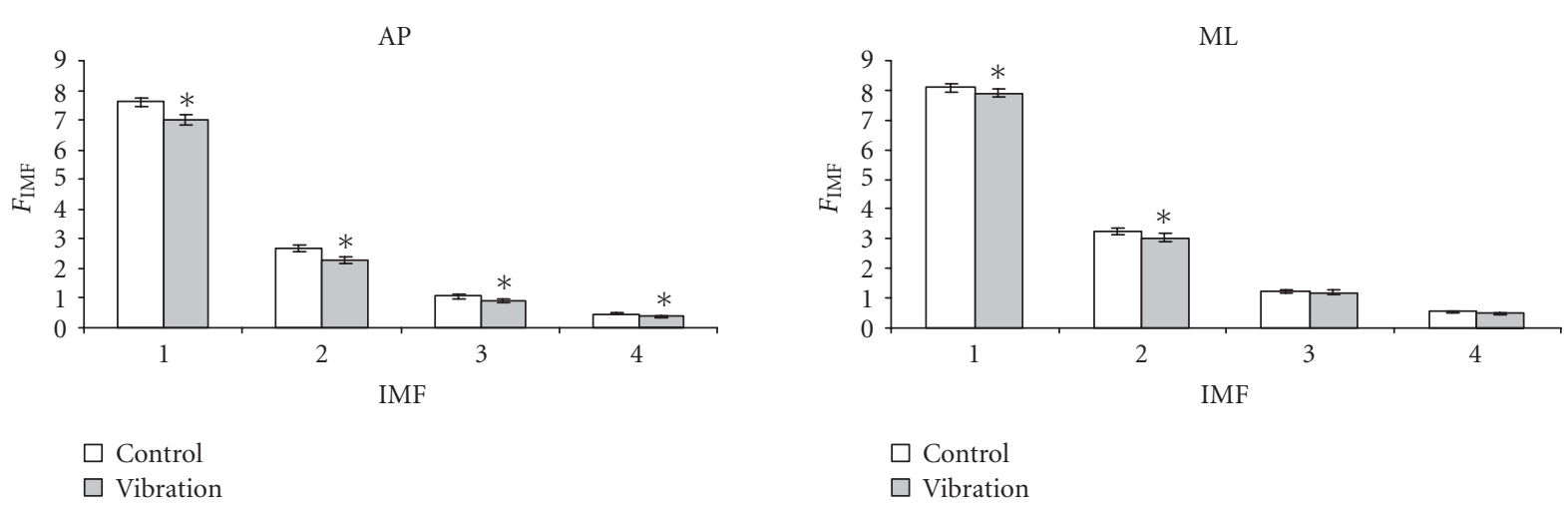

(a)

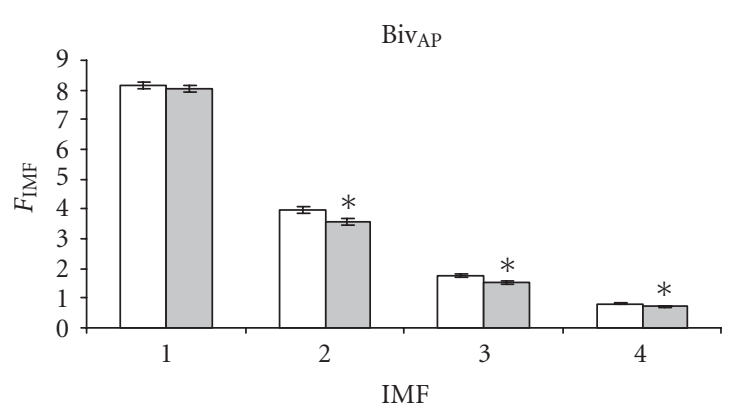

$$
\begin{aligned}
& \square \text { Control } \\
& \square \text { Vibration }
\end{aligned}
$$

(b)

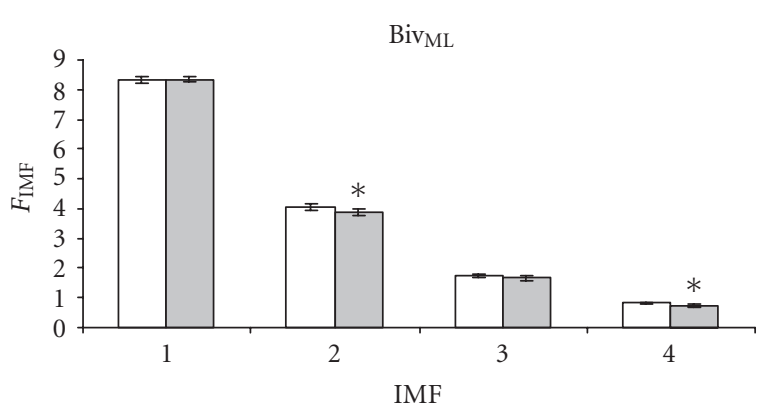

$$
\begin{aligned}
& \square \text { Control } \\
& \square \text { Vibration }
\end{aligned}
$$

(c)

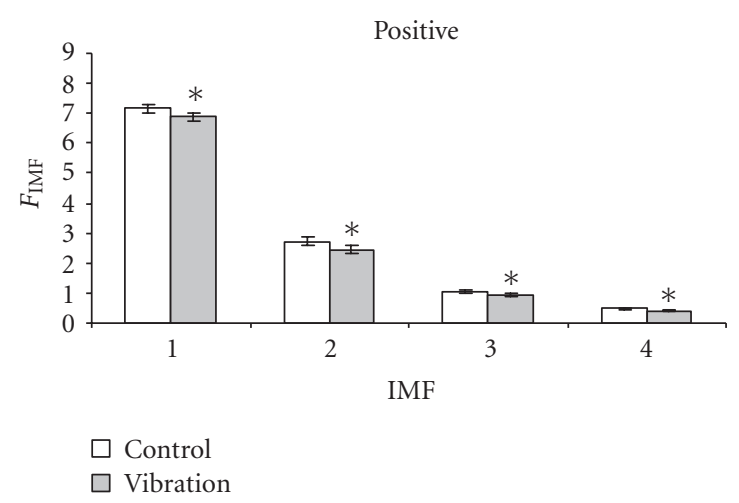

(e)

$$
\begin{aligned}
& \square \text { Control } \\
& \square \text { Vibration }
\end{aligned}
$$

(d)

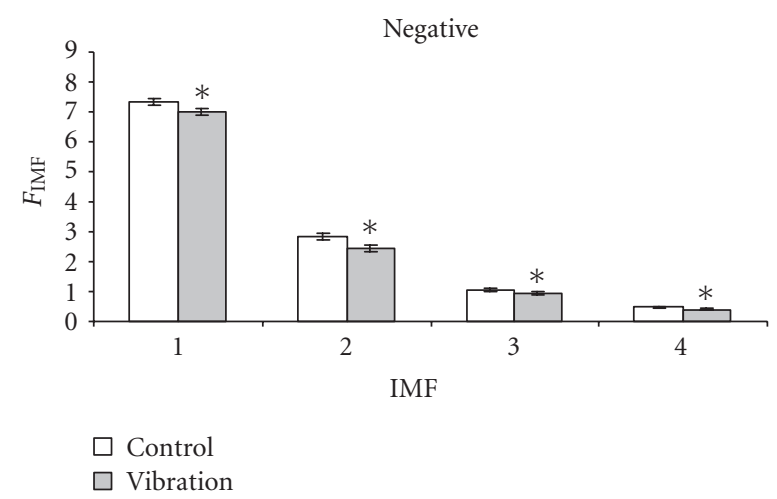

(f)

FIGURE 7: The average rotation frequency $\left(F_{\text {IMF }}\right)$ of the first four IMF for control and vibration subjects. (a) EMD for AP displacement; (b) EMD for ML displacement; (c) bivariate-EMD for the imaginary part of the signal (corresponding to AP displacement); (d) bivariate-EMD for the real part of the signal (ML); (e) complex-EMD for positive components; and (f) complex-EMD for negative components. Data are mean and $95 \%$ confidence intervals. ${ }^{*}$ Significant difference from control subjects.

stabilogram, AP and ML displacements are decomposed separately in the basic-EMD, despite the fact that the same postural control systems produce these two signals. It is almost certain that these two signals are linearly and/or nonlinearly correlated. In addition, physiological interpretation could be difficult as mutual information is lost.

The complex-EMD method decomposes the positive and negative components separately, as for two independent signals. The IMFs resulting from the positive and negative components form two independent sets of IMFs for which it is not necessary that the number of IMFs is equal for positive and negative components. However, interpretation of the positive and negative frequency is difficult as the positive and negative components bear no obvious relationship with physiological control systems. Despite this, complex-EMD was the only method that enabled discrimination between elderly and control groups using the average rotation frequency for all of the first four IMFs for both positive and negative frequency components. Confirmation of the capacity of the complex-EMD method to detect differences between groups based on average rotation frequencies was performed using data from an experimental study in which 
the proprioceptive and visual systems were degraded by applying tendon vibration, and by closing subjects' eyes. Details of the experiments performed on 17 healthy young subjects can be found in [16]. The same EMD, complexEMD, and bivariate-EMD analyses were applied on the data from these experiments. In respect to the area, similar results were obtained between groups, with greater surface areas and thus increased postural sway observed for vibration. In respect to the average rotation frequency, the complexEMD method again outperformed the two others, with significant differences observed between groups for all four IMF for both positive and negative rotation (Figure 7). In contrast, for the basic-EMD method, significant differences were observed for all IMFs for AP displacement and for the first and second IMFs for ML displacement. With respect to the bivariate-EMD method, only three of the IMFs for AP and two of the IMFs for ML showed significant differences between groups (Figure 7). Furthermore, the IMFs in which these differences were observed were not the same as those identified in Figure 6 for the control and elderly subjects.

In addition, we have studied the influence of different values of $M$ (from $M=4$ to $M=16$ ) on the ability of the bivariate-EMD to discriminate between groups. The statistical significance of results is unaltered for the surface parameter $\mathrm{Area}_{\mathrm{IMF}}$ for the different values of $M$. However, varying the value of $M$ may cause the change of the statistical significance of the average rotation frequency to discriminate between groups. The statistical significance is lost for certain IMFs and may appear for other IMFs while varying the value of $M$.

The difference between results is not surprising for the bivariate-EMD method. This method is based on the rotation in two-dimensional space, while the extraction of extrema is related to the change of the moving point direction [15]. Due to this fundamental difference in methodology, the results for the average rotation frequency are not the same for the bivariate-EMD.

In respect to the sifting process used by the different methods, complex-EMD uses the basic-EMD method. The only difference is that the complex-EMD decomposes the complex signal into two positive and negative frequency components before applying the sifting process.

Concerning the bivariate-EMD method, despite the less impressive results shown in the present study when compared to the complex-EMD method, bivariate-EMD might offer some advantages under specific conditions. For instance, rather than split the complex signal into two parts, the bivariate-EMD decomposition is done on the complex signal directly, thus the problem of the number of IMFs for the positive and negative frequency components does not arise due to the unified approach adopted to decompose the complex signal. In addition, the mutual dependency between the real and imaginary parts of the complex signal is taken into account. In this way, bivariate-EMD might be more likely to respond to changes in spatial parameters than the others methods. Furthermore, bivariate-EMD enables the extraction of the fast rotation that is superimposed on slower rotation in the COP signals. In this way, it could be possible to identify the characteristics of different posture control systems in future studies.

\section{CONCLUSION}

The EMD, complex-EMD, and bivariate-EMD methods are useful and powerful methods to analyze nonstationary univariate and bivariate time series. In the case of stabilogram time series, these methods were able to extract the oscillations in different adaptive time scales, and to define proper rotations that could be related to the different postural control systems. In addition, these methods enabled the quality of equilibrium of postural systems to be characterized as well as to identify the differences in postural control mechanisms between elderly and control subjects. In fact, the use of the different types of EMD methods enabled the extraction of individual centers of rotation for each IMF. In perspective, additional work is planned in order to find the relation between IMFs and the different postural control systems. A follow-up study will address this issue, initially by simulation, before using different experimental conditions in which the different postural control systems will be impaired. The aim of this study will be to ascertain whether or not each IMF corresponds to a given postural control system.

\section{ACKNOWLEDGMENTS}

This study was undertaken as part of the mv-EMD research project (multivariate Empirical Mode Decomposition) supported by the French ANR agency (ANR Blanc Grant BLAN07-1_223026), the PréDICA research project (Prévision, Détection Investigation contre la Chute des Personnes Âgées) supported by the French ANR agency (Grant ANR-O5-RNTS-01801), and the PARAChute research project (Personnes Agées et Risque de Chute) which was supported in part by The French Ministry of Research (Grant 03-B-254), The European Social Fund (Grant 3/1/3/4/07/3/3/011), The European Regional Development Fund (Grant 2003-2-50-0014 and Grant 2006-2-200011), The Champagne-Ardenne Regional Council (Grant E200308251), and INRIA (Grant 804F04620016000081).

\section{REFERENCES}

[1] Comité Français d'Education pour la Santé, "Les clés du "bien vieillir": prévention des chutes chez les seniors," Caisse Nationale de l'Assurance Maladie des Travailleurs Salariés, November 2001.

[2] "The 2005 epc projections of age-related expenditure (200450) for the eu- 25 member states," Tech. Rep. 4, European Commission, Luxembourg, 2005.

[3] L. Z. Rubenstein and K. R. Josephson, "The epidemiology of falls and syncope," Clinics in Geriatric Medicine, vol. 18, no. 2, pp. 141-158, 2002.

[4] T. E. Prieto, J. B. Myklebust, R. G. Hoffmann, E. G. Lovett, and B. M. Myklebust, "Measures of postural steadiness: differences between healthy young and elderly adults," IEEE Transactions on Biomedical Engineering, vol. 43, no. 9, pp. 956-966, 1996. 
[5] T. E. Prieto, J. B. Myklebust, and B. M. Myklebust, "Characterization and modeling of postural steadiness in the elderly: a review," IEEE Transactions on Rehabilitation Engineering, vol. 1, no. 1, pp. 26-34, 1993.

[6] J. J. Collins and C. J. De Luca, "Upright, correlated random walks: a statistical-biomechanics approach to the human postural control system," Chaos, vol. 5, no. 1, pp. 57-63, 1995.

[7] H. Amoud, M. Abadi, D. Hewson, V. Michel-Pellegrino, M. Doussot, and J. Duchêne, "Fractal time series analysis of postural stability in elderly and control subjects," Journal of NeuroEngineering and Rehabilitation, vol. 4, pp. 1-12, 2007.

[8] H. Snoussi, H. Amoud, M. Doussot, D. Hewson, and J. Duchêne, "Reconstructed phase spaces of intrinsic mode functions. Application to postural stability analysis," in Proceedings of the 28th Annual International Conference of the IEEE Engineering in Medicine and Biology Society (EMBS '06), pp. 4584-4589, New York, NY, USA, August-September 2006.

[9] H. Amoud, H. Snoussi, D. Hewson, M. Doussot, and J. Duchêne, "Intrinsic mode entropy for nonlinear discriminant analysis," IEEE Signal Processing Letters, vol. 14, no. 5, pp. 297300, 2007.

[10] J. P. Carroll and W. Freedman, "Nonstationary properties of postural sway," Journal of Biomechanics, vol. 26, no. 4-5, pp. 409-416, 1993.

[11] G. F. Harris, S. A. Riedel, D. Matesi, and P. Smith, "Standing postural stability assessment and signal stationarity in children with cerebral palsy," IEEE Transactions on Rehabilitation Engineering, vol. 1, no. 1, pp. 35-42, 1993.

[12] N. E. Huang, Z. Shen, S. R. Long, et al., "The empirical mode decomposition and the Hilbert spectrum for nonlinear and non-stationary time series analysis," Proceedings of the Royal Society of London A, vol. 454, no. 1971, pp. 903-995, 1998.

[13] T. Tanaka and D. P. Mandic, "Complex empirical mode decomposition,” IEEE Signal Processing Letters, vol. 14, no. 2, pp. 101-104, 2007.

[14] G. Rilling, P. Flandrin, P. Gonçalves, and J. M. Lilly, "Bivariate empirical mode decomposition," IEEE Signal Processing Letters, vol. 14, no. 12, pp. 936-939, 2007.

[15] M. U. Bin Altaf, T. Gautama, T. Tanaka, and D. P. Mandic, "Rotation invariant complex empirical mode decomposition," in Proceedings of the IEEE International Conference on Acoustics, Speech and Signal Processing (ICASSP '07), vol. 3, pp. 1009-1012, Honolulu, Hawaii, USA, April 2007.

[16] V. Michel-Pellegrino, H. Amoud, D. Hewson, and J. Duchêne, "Identification of a degradation in postural equilibrium invoked by different vibration frequencies on the tibialis anterior tendon," in Proceedings of the 28th Annual International Conference of the IEEE Engineering in Medicine and Biology Society (EMBS '06), pp. 4047-4050, New York, NY, USA, August-September 2006. 